\title{
Ex- situ and in-situ microstructure investigations of AM Part and powders
}

\author{
M. Albu ${ }^{1}$, S. Mitsche ${ }^{2}$, M. Nachtnebel ${ }^{1}$, R. Krisper ${ }^{1}$, M. Dienstleder ${ }^{1}$, H. Schroettner ${ }^{2}$, \\ G. Kothleitner ${ }^{2}$
}

\begin{abstract}
This paper presents an advanced microstructural analysis of the AlSiMg, Ti64 and N700 powders used for additive manufacturing. The internal microstructure of the regular and irregular powder grains were characterized down to atomic resolution by using scanning electron microscopy and high resolution scanning transmission electron microscopy.

The accretionary forms on top of the irregular AlSiMg powder grains exhibit a slightly coarse microstructure with a network of eutectic Si consisting of nano-crystallites, suggesting a slower cooling than the grain itself that contain a predominately amorphous Si network. A nm thin amorphous $\mathrm{C}$ layer on the surface of some Ti64 plasma atomized powder grains promoted the attachment of satellites and growth of envelopes. In case of gas atomized N700 powder grains, we identified thin oxide and carbon amorphous layers as well as metal segregations at the interface between the grain body and the accretionary forms.
\end{abstract}

Keywords: Powder, AlSiMg, Ti64, N700, microstructure, high-resolution electron microscopy

\section{Introduction}

The mechanical properties, static and dynamic, of the final additive manufactured part are governed by several inputs and parameters that affect the microstructure of the printed part. Since most common systems used for additive manufacturing (selective laser melting and electron beam melting) use metal powders as feedstock, their characterization is of central importance. The quality of the powder is determined by size, surface morphology, composition and amount of internal porosity. Old and recycled metal powders tend to oxidize and collect moisture when exposed to air, therefore the built chamber atmosphere should contain a shield gas. However these gases my affect the chemistry, processability and the heat transfer, which, in turn, affects the microstructure of the printed part $[1,2,3,4]$.

Whereas the chemical composition, size, surface morphology and internal porosity have been well characterized by the producers, less attention has been given to the internal microstructure of the powder grains and the cause of irregularities on the surface of the grains (satellites and accretionary forms or envelopes) [5].

This paper attempts to provide insights into the origin of the irregularities and changes in the internal microstructure of the powder grains, highlighting the importance of powder quality to the microstructure of the finalpart.

\footnotetext{
${ }^{1}$ Graz Centre for Electron Microscopy, Steyrergasse 17, $8010 \mathrm{Graz}$

${ }^{2}$ Institute for Electron Microscopy and Nanoanalysis, TU Graz, Steyrergasse 17, 8010 Graz
} 


\section{Materials and methods}

The AlSi10Mg, Ti64 and N700 powder grains were first dispersed on an adhesive tape and investigated in the scanning electron microscope (SEM). Additionally, the powder was embedded in a resin (CALDOFIX), polished down to a couple of tents of micrometres and thinned by $\mathrm{Ar}$-ion milling at cryogenic temperature. For EBDS measurements, the embedded grains were polished by using diamante and aluminium suspensions as final polishingsteps.

Scanning transmission electron microscopy (STEM) investigations have been performed on focused in beam (FIB) prepared lamellae cut out of the regular powder grains and grains with satellites and accretionary forms. For the in-situ heating experiment the lamella was mounted on a MEMS heating chip (DENS solutions - Wildfire H+ DT, heating/quench rate $200^{\circ} \mathrm{C} / \mathrm{ms}$ and settling time $2 \mathrm{~s}$ ).

The additive manufactured samples presented in this work were built by using the SLM method. The samples for SEM investigations were cut and polished along (z-axis) and perpendicular (xy-plan) to the build axis.

SEM micrographs were acquired by using backscattering, secondary electron and inlens detectors. For the analytical and crystallographic investigations of the grains and grain boundaries, dispersive X-ray spectrometry and EBSD were involved.

High-resolution STEM investigations were carried out by using the FEI Titan G3 60300 at a beam voltage of $300 \mathrm{kV}$. The advanced instrumentation fitted to this microscope, allows for recording HR-STEM images with annular dark field (ADF), annular bright field $(\mathrm{ABF})$ and high angle annular dark field (HAADF) detectors. Detection of chemical composition of different phases was performed by using energy dispersive X-ray analysis (EDX) and electron energy loss analysis were used. For energy dispersive X-ray analysis the microscope is equipped with a FEI Super-X windowless silicon drift detector (Chemi-STEM technology) and for electron energy loss spectroscopy the setup includes a Dual EELS Quantum Gatan Imaging Filter (GIF) [6].

\section{Experimental results}

Morphology, chemical composition and particle size of the powders were first characterized in the scanning electron microscope (SEM). Figure 1 shows the micrographs of the AlSi10Mg, Ti64 and N700 powder grains, their size distribution and roundness factor. Some grains contain some $\mu \mathrm{m}$-large but also nm-sized pores. We observed a rather large size distribution and a high amount of satellites in the gas-atomized powders (AlSiMg and N700).

Therefore, such grains with satellites and accretionary forms were investigated by STEM. We observed that regardless of alloy composition and production, a nm-thin layer of amorphous Carbon is present at the interface between particle and irregularities.

In case of Al-alloy, the microstructure of the accretionary form is coarser than the microstructure of the grain body indicating slower cooling. Therefore, the eutectic $\mathrm{Si}$ in the accretionary form is mostly nano-crystalline while in the grain body is mostly amorphous. Figure 2a presents the FIB lamella cut from an irregular powder grain, with the dotted line denoting the interface between the grain body and accretionary form. Figures $2 \mathrm{~b}$ and $\mathrm{c}$ show the eutectic $\mathrm{Si}$ in the grain body at higher magnification and at atomic resolution, with the insets on $2 \mathrm{c}$ being the Fourier transform of the amorphous $\mathrm{Si}$ and crystalline a-Al. The chemical composition of the eutectic Si network along the arrow on fig. $2 \mathrm{~b}$ is presented in fig. 2d. Nanometre-sized $\mathrm{Si}(\mathrm{Mg})$ particles were also dispersed all over the Al matrix. This 
microstructure is generated by the high cooling rates during the gas atomisation process. Insitu heating measurements performed on FIB lamella revealed the temperature at which the amorphous Si starts to form nano-crystallites to be $140^{\circ} \mathrm{C}$. A full nano-crystallization of the $\mathrm{Si}$ network was achieved at $350^{\circ} \mathrm{C}$. With further increase of temperature, the Si network became coarse globular and monocrystalline.
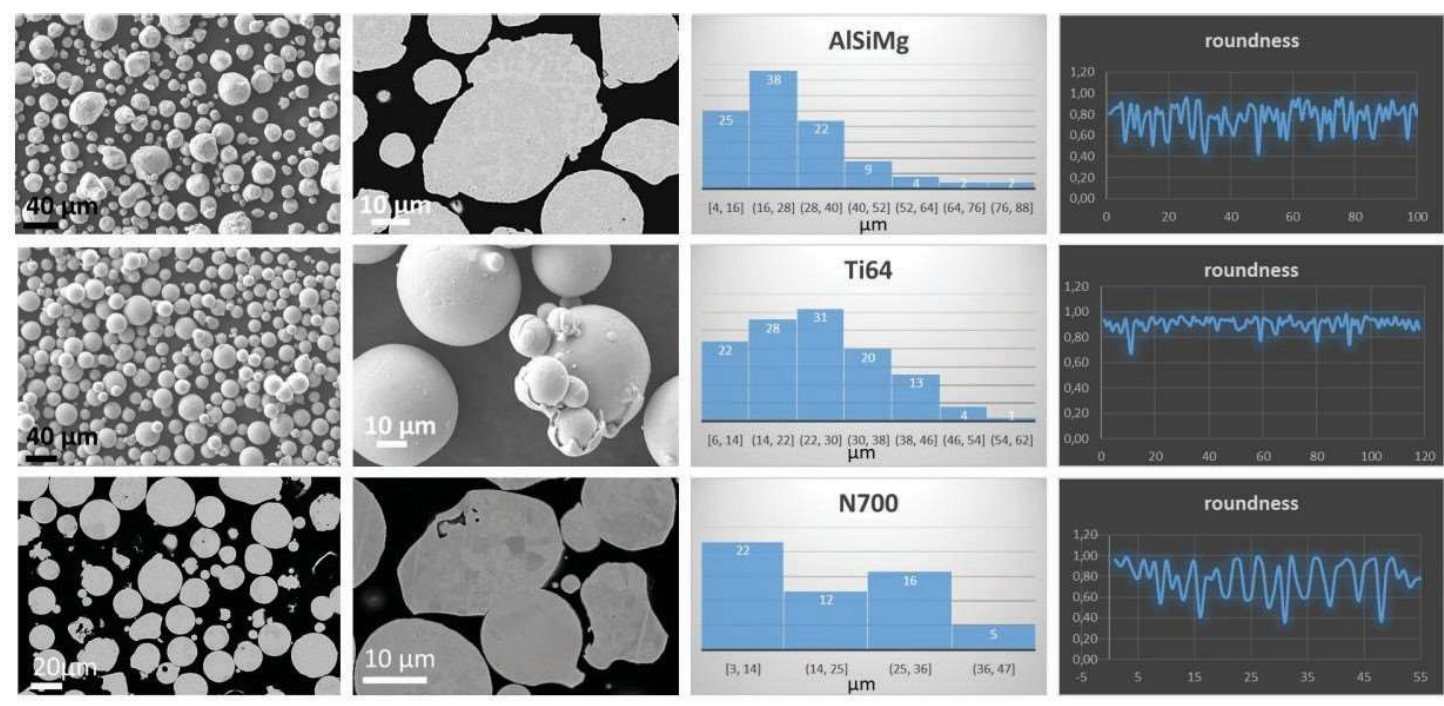

Fig. 1 SEM images, size distributions and roundness of the AlSi10Mg (top row), Ti64 (middle row) and N700 powders (bottom row).
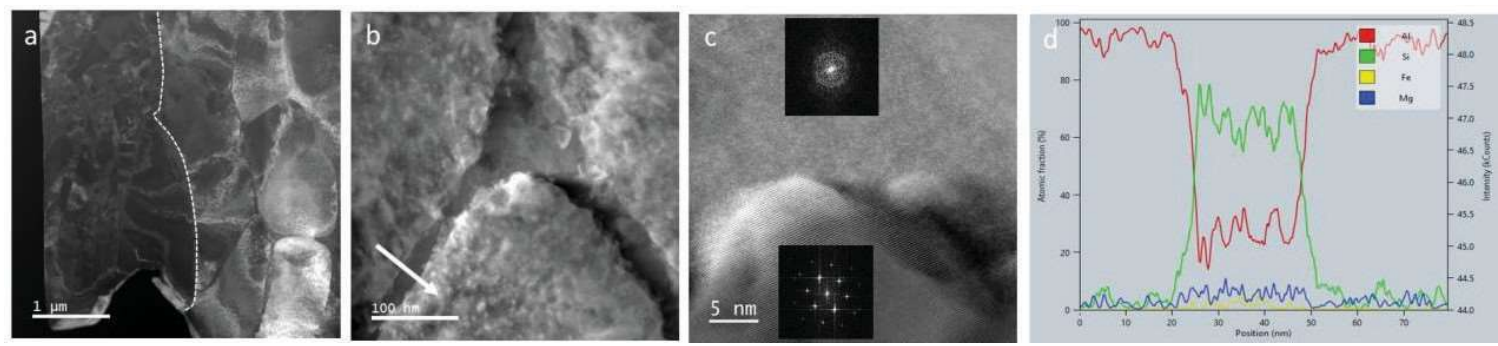

Fig. 2 a) STEM ADF image of the FIB lamella cut from an irregular powder grain, dotted line denotes the interface between grain body (left) and accretionary form (right); b) higher magnification STEM ADF image of the Si network; c) atomic resolution image of the amorphous eutectic Si. Insets show the FFT of the amorphous Si and the a-Al crystalline regions. d) EDX element profile along the arrow in b).

The as printed sample showed similar fine microstructure with nano-crystalized eutectic Si and nanometre-sized $\mathrm{Fe}$ segregations dispersed in the $\mathrm{Si}$ network. Moreover, $\mathrm{Si}(\mathrm{Mg})$ nanoparticles of about 5-10 $\mathrm{nm}$ were dispersed in the Al matrix. Similar observations have been made in other AlSi alloys [7, 8]. Fe-rich intermetallic phases were also observed at the boundary of the a-Al cells, intermixed within the Si network.

In order to observe the statistic distribution of the a- and B-phases within the plasma atomized Ti64 powder grains we performed EBDS measurements. The images in figure 3a 
and $3 \mathrm{~b}$ show the a- and the B-phase respectively. Some powder grains appear to not contain Bphase (probably in the nm range and therefore not detectable in SEM), whereas in others their fraction is very high.

The microstructure of the powder grains consists mainly of a- and B-phases. However, by using STEM on FIB lamella, we identified besides the B-phase with about 12.5 at $\%$ of V, small vanadium rich regions about $50 \mathrm{~nm}$ large (figure $5 \mathrm{a}$ ) with a ratio Ti/V of 1.5 (figure $5 \mathrm{~b}$ ), which were assigned to the $\mathrm{m}$ phase.
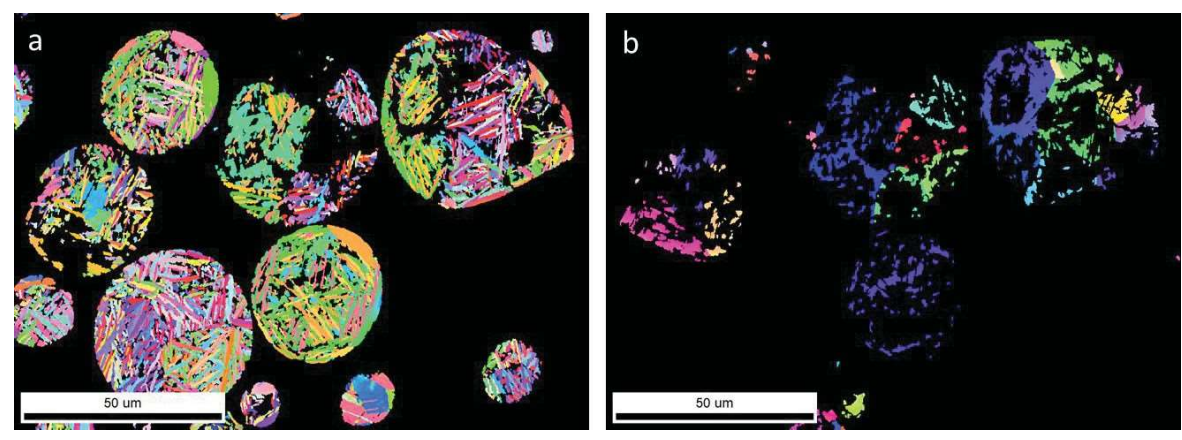

Fig. 3 EBSD images of a) a phase and b) B phase.

The SEM image of an irregular grain in Ti64 powder from which a FIB lamella was cut, is presented in figure $4 \mathrm{a}$ and $\mathrm{b}$. Figure $4 \mathrm{c}$ shows the STEM HAADF image of the lamella, and $4 \mathrm{~d}$ and $4 \mathrm{e}$ presents higher magnification STEM images at the interface between grain body and envelope. Ti, O, and C EELS elemental maps are presented in $4 \mathrm{~d}$ and the $\sim 20 \mathrm{~nm}$ amorphous $\mathrm{C}$ layer along the interface is also shown on the HR TEM image as inset of fig. 4e. The EELS chemical analysis of the amorphous layer on top of the particle situated between grain body and envelope indicates regions of amorphous $\mathrm{TiO}_{2}$ and amorphous $\mathrm{C}$.

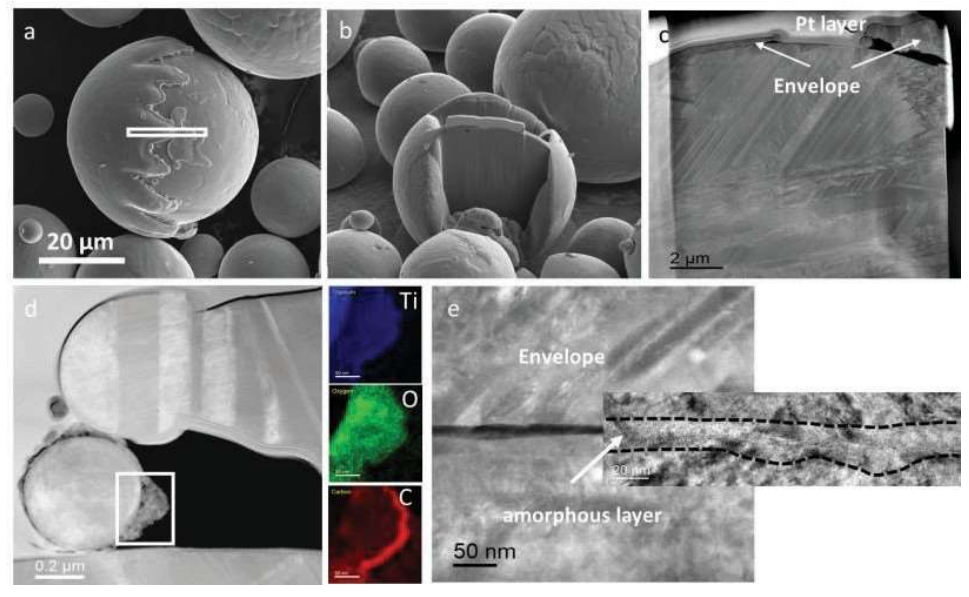

Fig. 4 a) SEM image of an irregular Ti64 powder grain from which the FIB lamella in b) was cut. c) STEM HAADF image of the lamella indicating the particle body and the envelope as well as the Pt layer deposited during the FIB process; d) higher magnification STEM HAADF image of the particle incorporated into the envelope and the chemical analysis (EELS 
spectrum image) of the amorphous layer on top of it. e) high magnification STEM image of the interface between grain body and envelope with a high-resolution TEM image of the amorphous Carbon as inset.
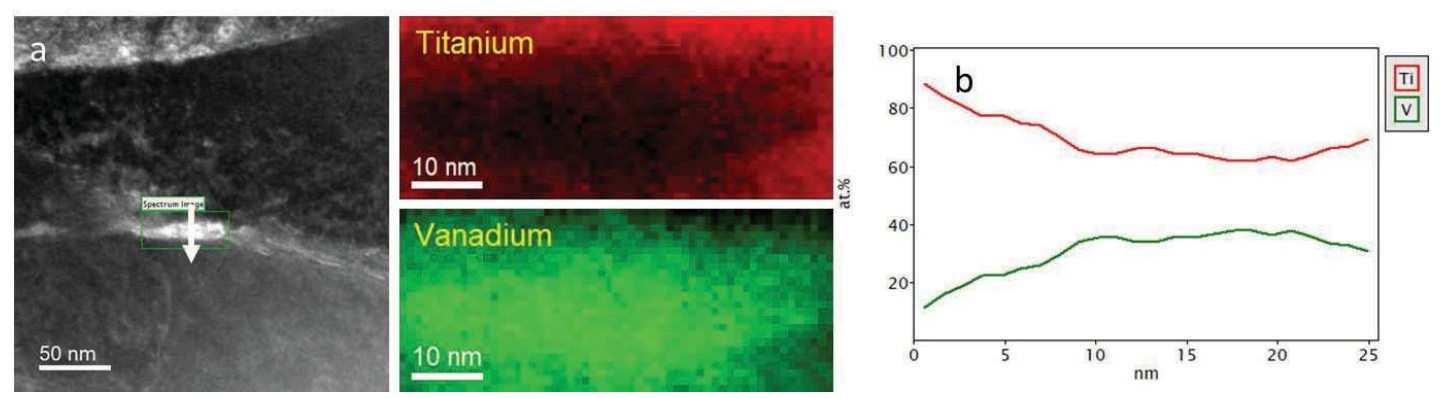

Fig. 5 a) STEM HAADF image of a lamella cut from a regular Ti64 powder grain containing $\mathrm{m}$ phase and the EELS chemical maps of $\mathrm{V}$ and Ti. b) EELS element profile along the arrow drown on the investigated area showing the high content of $\mathrm{V}(\sim 40 \mathrm{at} \%)$.

The as printed sample exhibits a fine microstructure consisting of acicular B phase mixed with a fine-lamellar a-phase, as observed on the SEM and EBSD images in figures 6 and 7. The chemical composition of the V-rich phase in figure $5 \mathrm{~b}$ detected by EDX, shows concentrations of $16-18 \mathrm{wt} \%$ of $\mathrm{V}, 4.5-4 \mathrm{wt} \%$ of Fe, and $4.2-5.4 \mathrm{wt} \%$ of Al. The B-phase (fig. 7b) with dimensions between $200 \mathrm{~nm}$ and $6 \mu \mathrm{m}$ is evenly distributed all over the sample, with its fraction in the as printed sample being about 3\%. At such high cooling rate, the aphase lamellae are, as expected, partially basket-like arranged.
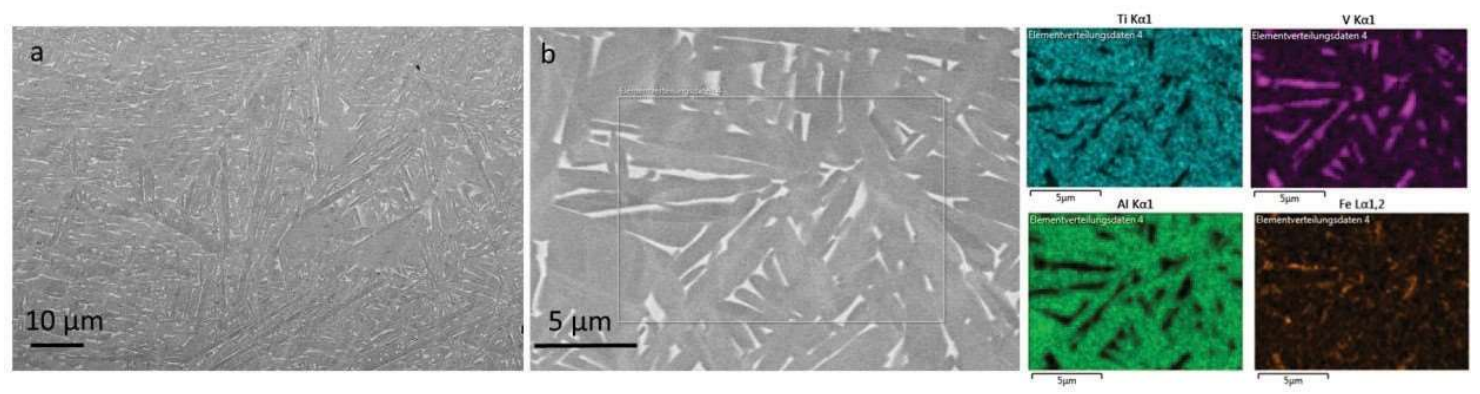

Fig. 6 SEM images at a) low and b) higher magnification together with the EDX chemical analysis of the as-built Ti64 sample.
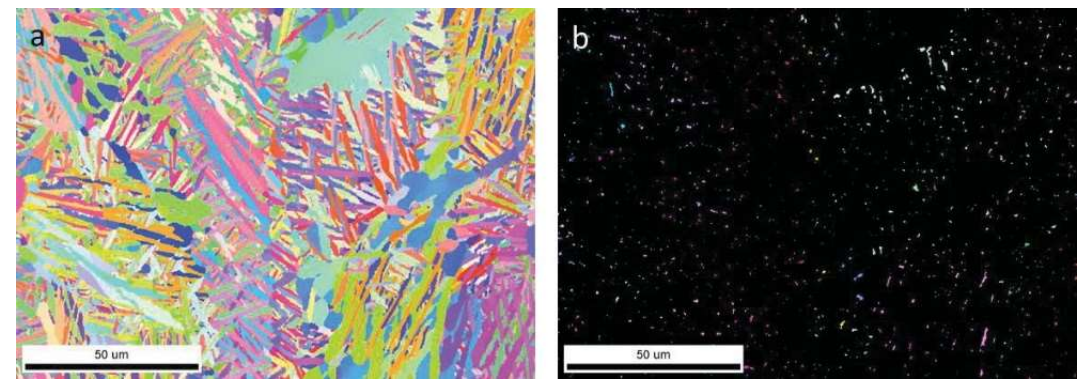

Fig. 7 EBSD images of the as-built Ti64 sample (build axis): a) a-phase and b) B-phase.

The N700 powder was produced by gas atomization (BÖHLER N700 AMPO [9]). As observed on figure 1 bottom row, the powder presents a low roundness factor with a large 
size distribution $(14-45 \mu \mathrm{m})[10]$.

The intrinsic microstructure of a regular powder grain (fig. 8) is homogenous with no nanometre-sized precipitates and the electron diffraction investigation (fig. 8e) indicates the structure of the a-ferrite (bcc) with a lattice parameter of $0.2885 \mathrm{~nm}$. The lattice parameter of a-ferrite in powders can increase from $0.2866 \mathrm{~nm}$ up to $0.29199 \mathrm{~nm}$ with increasing temperature as described in literature [11].
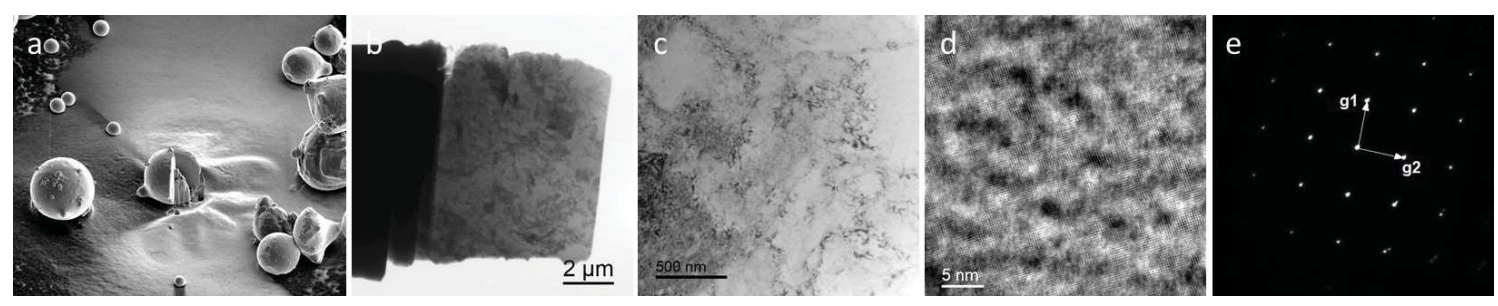

Fig. 8 a) SEM image of a regular N700 powder grain from which the lamella in b) was cut. c) higher magnification TEM zero loss filtered image of the lamella in b); d) high resolution TEM image from the lamella; e) SAED electron diffraction image from the region in c) with $\mathrm{d}_{1}=\mathrm{d}_{2}=0.2885 \mathrm{~nm}$.

Figure 9 illustrates the analysis of a lamella cut out of an irregular grain in the N700 powder. We observed two different amorphous layers at the interface between grain body and envelope: oxygen (iron oxide of about $10 \mathrm{~nm}$ thick) and carbon $(5-8 \mathrm{~nm})$, but also a metallic $\mathrm{Cu}$ layer around $10 \mathrm{~nm}$ thick. The $\mathrm{Cu}$ segregation is rather unexpected but not impossible since the alloy contain 3-5 wt $\%$ of $\mathrm{Cu}$ and the cooling rate during production is very high.

The SLM as printed part of N700 steel presents a fine ferrite microstructure with lamellar morphology about $1-20 \mu \mathrm{m}$ large, and very small grains of austenite $(<1.5 \mu \mathrm{m})$. The fine microstructure can be observed on the back scattered SEM images at higher magnification, in the built direction (fig. 10a) and perpendicular to the build direction (fig. 10d). The EBSD images illustrates the orientation of ferrite (figs. 10b and e) and austenite phases (figs. 10c and f) in the built direction and perpendicular to the build direction, respectively. 

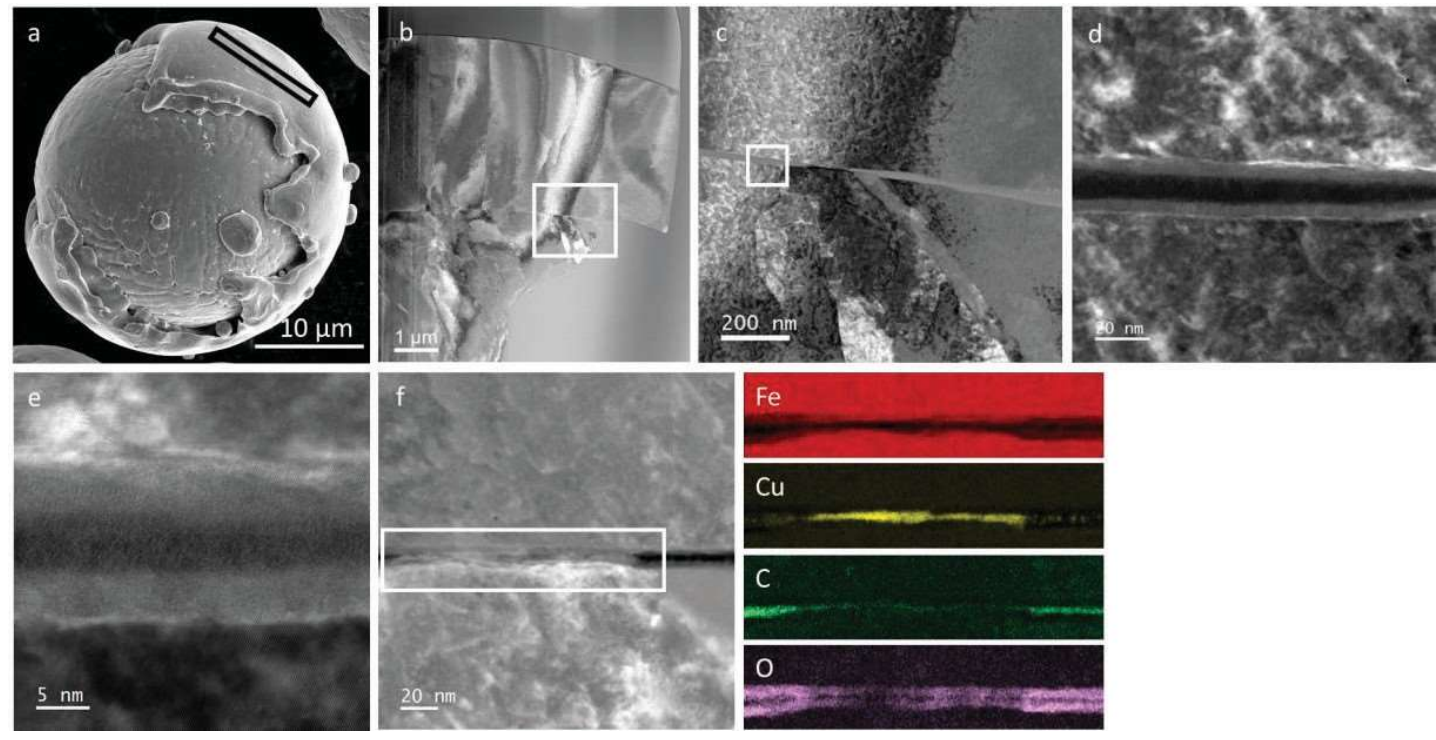

Fig. 9 a) STEM HAADF image of the lamella cut out from an irregular N700 powder grain. b) Higher magnification from the region indicated with a square in a; c) and d) high resolution images from the left square on image b) showing the interface between the grain body and the envelope - with two different amorphous layers; e) high resolution image of the right square in b), and f) HAADF reference image for the EDX spectrum image from which the elemental maps of $\mathrm{Fe}, \mathrm{Cu}, \mathrm{C}$ and $\mathrm{O}$ were extracted.
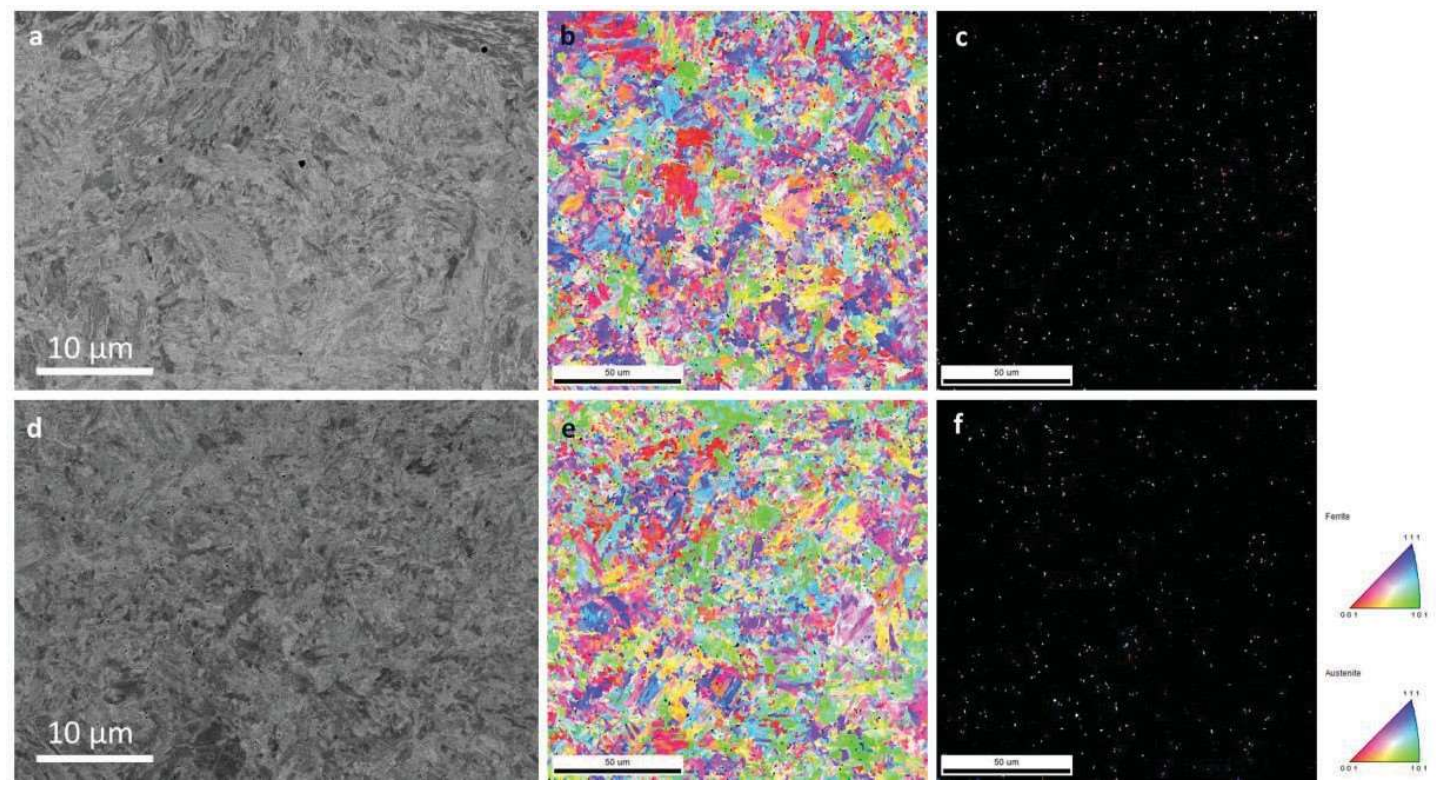

Fig. 10 a) SEM image of the as printed N700 sample in the built direction (z); b) EBSD image of the ferrite phase in the built direction; c) EBSD image of the austenite phase the built direction; d) SEM image of the as printed N700 sample perpendicular to build direction (xy plane); e) EBSD image of the ferrite phase in the xy plane; f) EBSD image of the austenite phase in the xy plane. 


\section{Conclusions}

The understanding of microstructure change and evolution, correlated with the desired mechanical properties of the as-fabricated AM parts is strongly linked with the feedstock quality and AM process parameters. In order to reduce production and post-processing costs, powder characteristics and effects that might influence its quality, and therefore the quality of the as fabricated part, have to be well understood. Here, we presented detailed analyses of the internal microstructure of powder grains and the reason for irregularities on their surface.

The microstructure of the AlSiMg grains contains ultrafine cellular a-Al structures with a network of eutectic silicon predominantly amorphous in the gas-atomized powder. A partial nano-crystallization of the amorphous silicon was observed during in-situ heating experiment starting with $140^{\circ} \mathrm{C}$ and reaching the full crystallization at $350^{\circ} \mathrm{C}$. The accretionary forms on the surface of the powder grains, present a slightly coarser microstructure with a crystalline Si network, suggesting a slower cooling during the time of flight.

The Ti64 grains show a higher roundness factor, although satellites and envelopes are also attached to the surface of some grains. Their existence seems to be linked to the presence of a $20 \mathrm{~nm}$ amorphous $\mathrm{C}$ layer, situated at the interface between the satellites and envelopes with the grain body. The microstructure consists of a and B-phases, which are statistically distributed within the grains, but a sporadic occurrence of the m-phase, was also confirmed.

The satellites and accretionary forms are numerous in the N700 powder and linked to the presence of a $10 \mathrm{~nm}$ thick oxygen and a $5 \mathrm{~nm}$ thick carbon layer in amorphous phase, at the interface with the grain body. Moreover, there is a chance to find also other metal segregations such as $\mathrm{Cu}$.

\section{Acknowledgement}

We gratefully acknowledge our project partners Mr. B. Panzirsch and Mr. G. Schindelbacher from ÖGI, Leoben, Austria, for sample provision. The authors acknowledge financial support by the Austrian Science Fund (FFG): SP2018-003-006 (Microstructure of 3D printed metallic parts).

\section{References}

[1] Gibson, I., Rosen, D. W., and Stucker, B., Additive Manufacturing Technologies: 3D Printing, Rapid Prototyping, and Direct Digital manufacturing, 2nd., Springer, New York, (2015), pp. 1-498.

[2] H.P. Tang, M. Qian, N. Liu, X.Z. Zhang, G.Y. Yangand, J. Wang, JOM (2015), 1 -9.

[3] J. A. Slotwinski, E. J. Garboczi, P. E. Stutzman, C. F. Ferraris, S. S. Watsonand, M.A. Peltz, J. Res Natl. Inst. Stand. Technol. (2014), 119

[4] B. Chen, S.K. Moon, X. Yao, G. Bi, J. Shen, J. Umeda, K. Kondoh, Scripta Mater. (2015, Volume 141), 45-49

[5] L. Zhou, A. Mehta, E. Schulz, B. McWilliams, K. Cho, Y. Sohn, Mat. Charact. (2018, Volume 143), 5-17

[6] A. Gubbens, M. Barfels, C. Trevor, R. Twesten, P. Mooney, P. Thomas, N. Menon, B. Kraus, C. Mao, B. McGinn, Ultramicroscopy (2010, Volume 110) 962-970.

[7] M. Liu, H. Fu, L. Tian, W. Xiao, Q. Peng, C. Ma, Materials and Design, (2017, Volume 121), 373-382 
[8] X.P. Li, G. Ji, Z. Chen, A. Addad, Y. Wu, H.W. Wang, J. Vleugels, J. Van Humbeeck, J.P. Kruth, Acta Mater. (2017, Volume 129), 183-193

[9] voestalpine BÖHLER Edelstahl GmbH \& Co KG, BÖHLER N700 AMPO Product Data Sheet, 2018

[10] C. Turk et al., Berg Huettenmaenn Monatsh (2019, Volume 164 / 3), 112-116 https://doi.org/10.1007/s00501-019-0835-z

[11] Zhiyao Feng, Master Thesis, "The lattice parameter of gamma Iron and Iron chromium alloys", Dept. Mat. Sci and Eng., Case Western Reserve University, May 2015 